
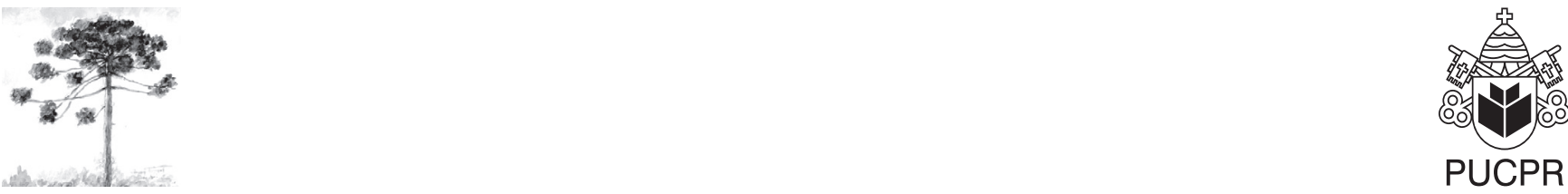

PUCPR

\title{
Estrutura populacional, hábitos alimentares e aspectos reprodutivos de Charax stenopterus (COPE, 1894) (Teleostei, Characidae): uma espécie introduzida no reservatório do Passaúna, Sul do Brasil
}

\author{
Population structure, feeding habits and reproductive aspects of Charax \\ stenopterus (Cope, 1894) (Teleostei, Characidae): an introduced species in \\ Passaúna Reservoir, Southern Brazil
}

\author{
Vinícius Abilhoa ${ }^{[a]}$, Leticia Cristine de Lima ${ }^{[\mathrm{a}, \mathrm{c}]}$, Maria Ângela Plahtyn Torres ${ }^{[\mathrm{b}]}$, \\ Pâmela Regina Bastos Valério ${ }^{[\mathrm{c}]}$
}

[a] Biólogo, Doutor, Grupo de Pesquisas em Ictiofauna - Museu de História Natural Capão da Imbuia, Prefeitura de Curitiba, Curitiba, PR, Brasil, e-mail: vabilhoa@uol.com.br

[b] Licenciatura em Ciências Biológicas, Pontifícia Universidade Católica do Paraná (PUCPR), Curitiba, PR - Brasil.

[c] Bióloga, Grupo de Pesquisas em Ictiofauna - Museu de História Natural Capão da Imbuia, Prefeitura de Curitiba, Curitiba, PR Brasil, e-mail: ledelima@hotmail.com

\section{Resumo}

Neste trabalho foram investigados a estrutura populacional, os hábitos alimentares e a reprodução do lambari-transparente Charax stenopterus no Reservatório do Passaúna. Amostras mensais foram obtidas com redes de espera, entre julho de 2006 e maio de 2007. A estrutura populacional não apresentou variação nas médias de comprimento total e peso. Exemplares com gônadas maduras e em maturação ocorreram em todo o período amostral, com maior frequência de indivíduos maduros no verão, enquanto que gônadas imaturas e em descanso tiveram pouca representatividade. A análise da dieta, realizada por meio dos métodos de frequência de ocorrência, pontos e índice de importância alimentar, revelou uma pequena variedade de itens: microcrustáceos, estágios imaturos aquáticos de insetos, fragmentos de insetos terrestres, peixes e restos de vegetais. Os itens que tiveram maior representatividade foram peixes e larvas e pupas de Diptera. Considerando-se o pequeno porte, a estratégia reprodutiva e o espectro alimentar, esta espécie pode ser caracterizada como oportunista. Esta constatação indica que medidas de manejo devem ser planejadas, visando ao controle e monitoramento das populações introduzidas.

Palavras-chave: Alimentação. Espécie invasora. Lambari-transparente. Reprodução. 


\begin{abstract}
In this paper the population structure, reproduction and feeding habits of the glass-headstander Charax stenopterus in Passaúna Reservoir (Southern Brazil) were investigated. Monthly samples were made through gillnets, from July 2006 to May 2007. Significant differences in length and weigh mean values weren't found. Fishes in reproductive activities occurred in all study period, with high frequencies of mature individuals in summer. Gonads in immature and rest stages weren't representative. The diet analysis, assessed by the frequency of occurrence, point and alimentary index methods, revealed a small number of alimentary items: microcrustaceans, aquatic immature insects (larvae and pupae), terrestrial insect fragments, fish and plant fragments. Fishes and aquatic immature dipterans were the most representative items. Considering the small size, the reproductive strategy and the alimentary spectrum, the glass-headstander Charax stenopterus can be characterized as opportunistic. Because of that, management measures should be planned, in order to control and monitoring these introduced populations.
\end{abstract}

Keywords: Feeding. Glass headstander. Invasive species. Reproduction.

\section{Introduçáo}

O lambari-transparente Charax stenopterus (Cope, 1894) atinge cerca de $10 \mathrm{~cm}$ e apresenta ampla distribuição na América Latina, com registros nas bacias hidrográficas do Paraguai e Uruguai (1). No Brasil, sua distribuição original limita-se à Bacia do Rio Jacuí, lagoas e lagunas costeiras no Rio Grande do Sul e à Bacia do Prata, não ocorrendo no Rio Paraná acima de Sete Quedas, hoje inundada pelo reservatório de Itaipu (2).

Em coletas recentes realizadas no Reservatório do Rio Passaúna, localizado nas cabeceiras do Rio Iguaçu, na região metropolitana de Curitiba, esta espécie foi registrada com frequência (3), e sua ocorrência na região está relacionada provavelmente à sua introdução acidental ou intencional.

A presença desta espécie introduzida no reservatório pode reduzir ou extinguir as populações naturais locais, em virtude da competição por alimento, abrigo ou a disseminação de parasitos (4). Por este motivo, torna-se necessária a obtenção de informações sobre os aspectos da história natural de $C$. stenopterus neste reservatório, para que estratégias de conservação deste ecossistema sejam elaboradas.

\section{Material e métodos}

Os exemplares de C. stenopterus foram obtidos por meio de coletas mensais realizadas no Reservatório do Passaúna, localizado na região metropolitana de Curitiba, entre julho de 2006 e maio de 2007. As coletas foram feitas utilizando-se redes de espera com malhas simples de tamanhos $1 \frac{1}{2} 2,2,3 \frac{1}{2}$ e $4 \mathrm{~cm}$ entre nós consecutivos. Em campo, os peixes capturados foram etiquetados, ensacados, resfriados em isopor com gelo e levados ao Laboratório de Ictiologia do Museu de História Natural Capão da Imbuia. Em laboratório, os exemplares foram mensurados para a obtenção do comprimento total e padrão (com auxílio de régua milimetrada), pesados (com auxílio de balança de precisão de centésimo de grama) e dissecados para remoção do trato digestório, observação do sexo e do estádio de maturação gonadal macroscópico, segundo escala proposta em Vazzoler (5). As gônadas foram removidas e pesadas para o cálculo do índice gonadossomático (IGS), definido como a percentagem de participação do peso da gônada em relação ao peso total.

Para a análise dos dados de estrutura populacional em tamanho, a distribuição de frequência das classes de comprimento total para ambos os sexos foi obtida pela regra de Sturges (6). O período reprodutivo foi estimado a partir das distribuições das frequências dos estádios de desenvolvimento gonadal e do índice gonadossomático (5).

Os conteúdos estomacais foram analisados sob microscópio estereoscópico, sendo que a identificação dos itens alimentares foi realizada com auxílio de bibliografia especializada e por meio da consulta a especialistas. Para a análise da importância dos itens alimentares identificados, foram utilizados os métodos 
de frequência de ocorrência (FO), que avalia o percentual de estômagos em que determinado item alimentar ocorre em relação ao total de estômagos analisados e o método de pontos $(\mathrm{P})$, em que a contribuição de cada item foi determinada pela proporção de quadrículas ocupadas pelo item em uma superfície plana quadriculada em relação ao número total de quadrículas obtidas para todos os itens $(7,8)$. A integração dos dois métodos escolhidos para a análise da alimentação foi realizada por meio do Índice Alimentar (IAi) proposto por Kawakami e Vazzoler (9).

A tabulação dos dados, análises e confecção dos gráficos foram realizadas com o auxílio de planilha eletrônica (Microsoft ${ }^{\circledR}$ Office Excel 2003) e programa de análises estatísticas (Statistica ${ }^{\circledR}$ 6.0). Os testes estatísticos (ANOVA) foram realizados com emprego do programa Statistica ${ }^{\circledR}$ 6.0, e as diferenças implicaram nível de significância de 5\%.

\section{Resultados}

Os 189 exemplares de C. stenopterus capturados não apresentaram variações significativas nas médias de comprimento total $(\mathrm{F}=1,204$; $\mathrm{p}>$ $0,05)$ e peso $(F=1,359 ; p>0,05)$ entre as estações do ano (Tabela 1). A amplitude de tamanho variou da classe 8,5 a $9,0 \mathrm{~cm}$ à classe 11,1 a $12,0 \mathrm{~cm}$, e os indivíduos entre 9,1 e 9,5 cm foram mais frequentes nas capturas $(>30 \%$ ) (Tabela 2$)$. Mais da metade dos exemplares capturados encontravam-se entre as três primeiras classes de comprimento (entre 8,5 e 10,0 cm) (Gráfico 1).

Tabela 1 - Valores médios e desvios-padrão do comprimento total e peso dos exemplares de $C$. stenopterus capturados no Reservatório do Passaúna (2006/2007)

\begin{tabular}{lcc}
\hline Estações do ano & $\begin{array}{c}\text { Comprimento } \\
\text { total }(\mathbf{c m})\end{array}$ & Peso (g) \\
\hline Inverno & $10,1 \pm 0,9$ & $8,7 \pm 2,87$ \\
Primavera & $9,8 \pm 0,7$ & $7,8 \pm 1,98$ \\
Verão & $9,6 \pm 0,6$ & $8,1 \pm 1,52$ \\
Outono & $9,7 \pm 0,6$ & $6,9 \pm 1,12$ \\
\hline
\end{tabular}

Fonte: Dados da pesquisa.
Tabela 2 - Número (n), frequência relativa (\%) e estádios de maturação gonadal registrados em nas classes de comprimento total de $C$. stenopterus capturados no Reservatório do Passaúna (2006/2007)

\begin{tabular}{lcc}
\hline $\begin{array}{l}\text { Classes de comprimento } \\
\text { total }(\mathbf{c m})\end{array}$ & $\mathbf{n}$ & $\begin{array}{c}\text { Frequência } \\
\text { relativa } \mathbf{( \% )}\end{array}$ \\
\hline $1-8,5$ até 9,0 & 17 & 17,89 \\
$2-9,1$ até 9,5 & 31 & 32,63 \\
$3-9,6$ até 10,0 & 18 & 18,96 \\
$4-10,1$ até 10,5 & 13 & 13,68 \\
$5-10,6$ até 11,0 & 8 & 8,42 \\
$6-11,1$ até 12,0 & 8 & 8,42 \\
\hline
\end{tabular}

Fonte: Dados da pesquisa.

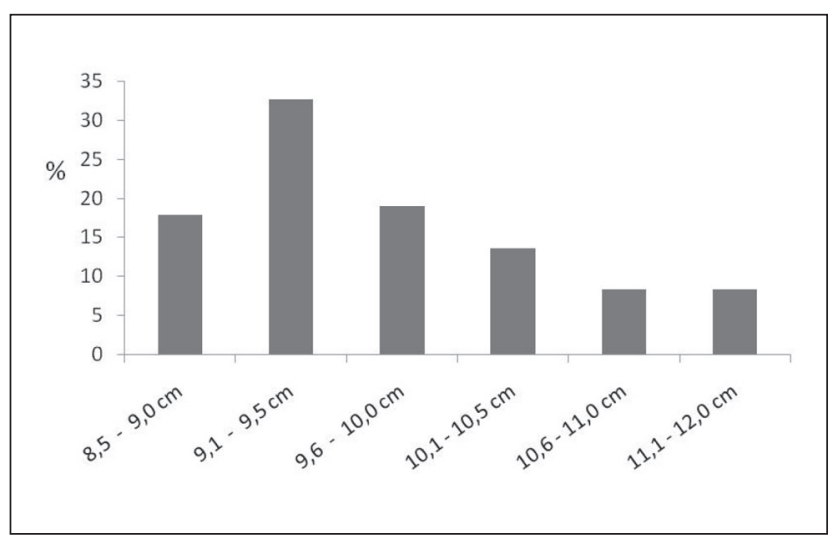

Gráfico 1 - Frequência dos indivíduos em cada classe de comprimento total (cm) de C. stenopterus capturados no reservatório do Passaúna (2006/2007)

Fonte: Dados da pesquisa.

As distribuições de frequência dos estádios de desenvolvimento gonadal foram ilustradas nos Gráficos 2 e 3. Exemplares com gônadas em maturação e maduras ocorreram em todo período amostral e em todas as classes de comprimento amostradas, enquanto que gônadas imaturas e em descanso tiveram pouca representatividade.

O período reprodutivo da espécie parece ser longo, com maior frequência de indivíduos maduros no verão (Gráfico 2). Os valores médios do índice 
gonadossomático para ambos os sexos não diferiram entre os meses amostrados ( $F=1,228$; $p>0,05)$, muito embora maiores valores tenham sido observados na primavera e verão (Grafico 4).

A frequência de ocorrência e o índice alimentar dos itens identificados na dieta de C. stenopterus foram listados na Tabela 3. Na Tabela 4 podem ser observadas as frequências de ocorrência e o índice alimentar dos itens organizados de acordo com as classe de comprimento total consideradas. A análise revelou uma pequena variedade de itens, os quais foram organizados em sete categorias. Os itens que tiveram maior representatividade foram larvas e pupas de Diptera (insetos) e peixes. Os itens de maior frequência durante o período amostral foram dípteros imaturos, sendo que este resultado somente não foi observado na primavera, em que a maior frequência e importância alimentar foram registradas para o item peixes. Com relação ao tamanho dos indivíduos, a frequência de ocorrência dos itens alimentares não apresentou grandes variações, sendo mais expressivos os itens larvas e pupas de Diptera e peixes.

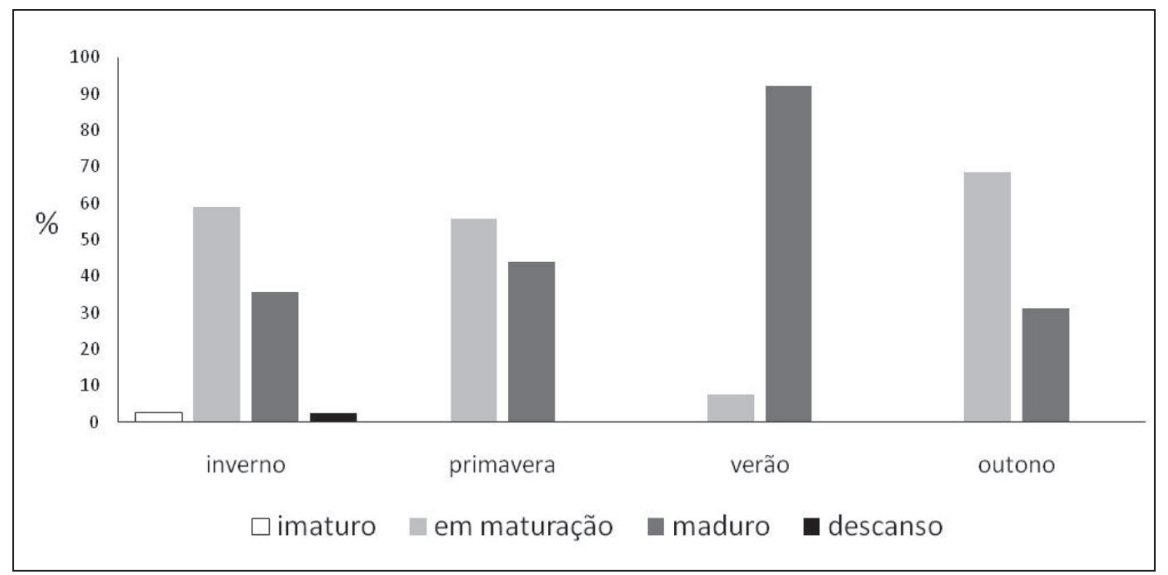

Gráfico 2 - Frequência dos indivíduos imaturos, em maturação, maduros e desovados de C. stenopterus capturados em cada estação do ano no reservatório do Passaúna (2006/2007)

Fonte: Dados da pesquisa.

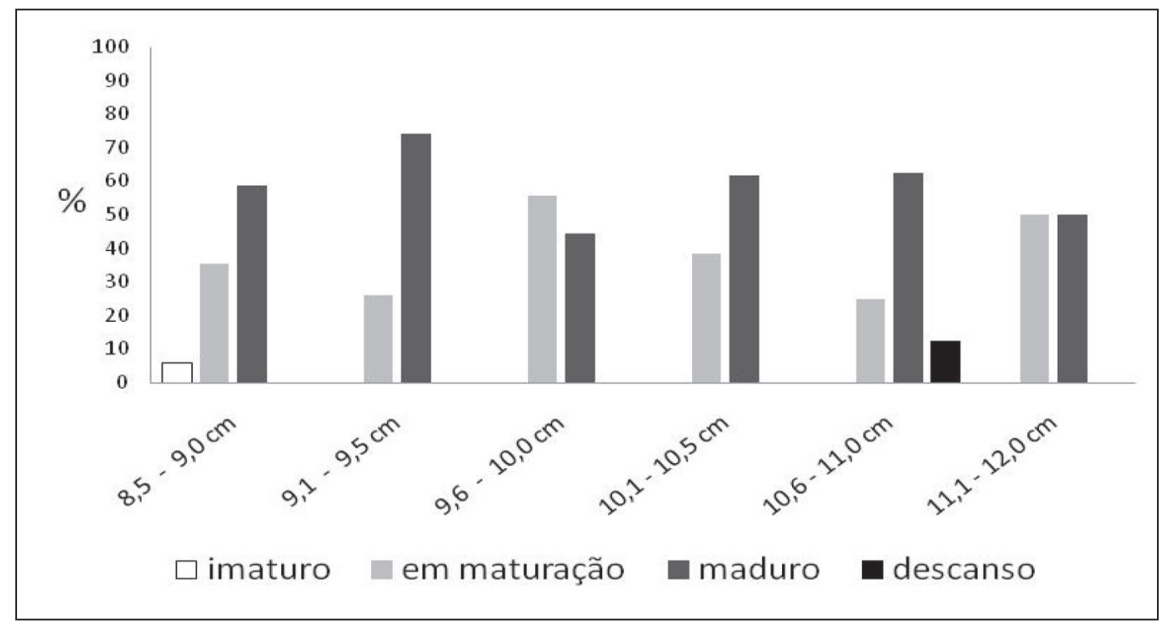

Gráfico 3 - Frequência dos indivíduos imaturos, em maturação, maduros e desovados de C. stenopterus em cada classe de comprimento total capturada no reservatório do Passaúna (2006/2007)

Fonte: Dados da pesquisa. 


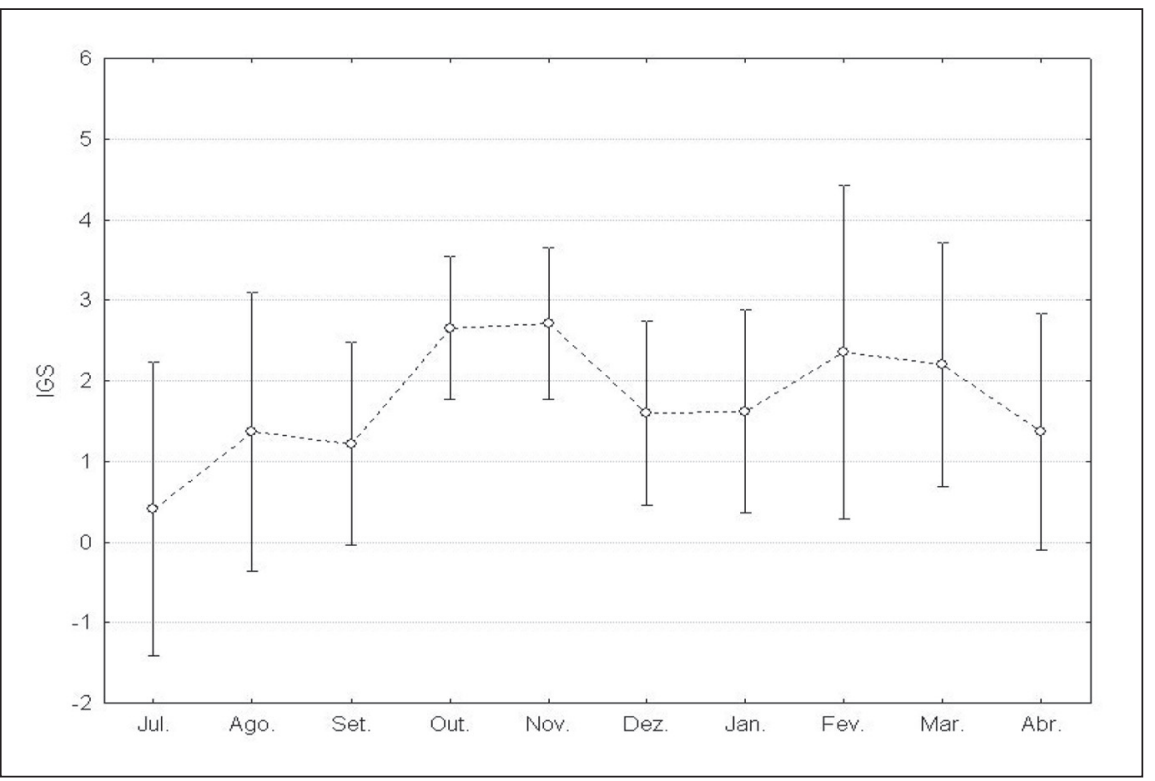

Gráfico 4 - Distribuição dos valores médios mensais do índice gonadossomático (IGS) de C. stenopterus capturados no reservatório do Passaúna $(2006 / 2007)$

Fonte: Dados da pesquisa.

Tabela 3 - Valores médios e desvios-padrão do comprimento total e peso dos exemplares de C. stenopterus capturados no Reservatório do Passaúna (2006/2007)

\begin{tabular}{|c|c|c|c|c|c|c|c|c|}
\hline \multirow[t]{3}{*}{ Itens alimentares } & \multirow{2}{*}{\multicolumn{2}{|c|}{$\begin{array}{l}\text { Inverno } \\
(\mathrm{n}=34)\end{array}$}} & \multirow{2}{*}{\multicolumn{2}{|c|}{$\begin{array}{c}\text { Primavera } \\
(\mathrm{n}=34)\end{array}$}} & \multirow{2}{*}{\multicolumn{2}{|c|}{$\begin{array}{c}\text { Verão } \\
(n=22)\end{array}$}} & \multirow{2}{*}{\multicolumn{2}{|c|}{$\begin{array}{l}\text { Outono } \\
(n=6)\end{array}$}} \\
\hline & & & & & & & & \\
\hline & FO & IA & FO & IA & FO & IA & FO & IA \\
\hline Material Vegetal & 8,8 & 0,7 & 14,7 & 0,69 & 4,54 & 0,07 & 16,6 & 0,2 \\
\hline Crustacea (Cladocera) & & & & & & & 16,6 & 0,05 \\
\hline Insecta - Diptera (larva/pupa) & 29,4 & 75,6 & 2,94 & 0,08 & 68,2 & 99,43 & 66,6 & 99,3 \\
\hline Insecta - Coleoptera (larva) & 2,9 & 5,6 & & & & & & \\
\hline Insecta - Trichoptera (larva) & & & 2,94 & 0,04 & & & & \\
\hline Fragmentos de insetos & 5,8 & 0,7 & 5,88 & 0,89 & & & 16,6 & 0,45 \\
\hline Peixes & 8,8 & 17,4 & 52,94 & 98,3 & 9,1 & 0,5 & & \\
\hline
\end{tabular}

Fonte: Dados da pesquisa.

\section{Discussáo}

Os motivos que levaram à introdução do lambari-transparente no Reservatório do Passaúna não são conhecidos. É provável que sua introdução tenha acontecido de maneira acidental, relacionada à aquicultura. De fato, a aquicultura é considerada o principal mecanismo de dispersão de espécies de peixes para novos ambientes - mais de $40 \%$ das introduções de peixes ocorreu por esta via (10). As 
Tabela 4 - Frequência de ocorrência (FO) e índice de importância alimentar (IA) dos itens registrados nos estômagos de C. stenopterus capturados no Reservatório do Passaúna (2006/2007), organizados de acordo com as classes de comprimento consideradas

\begin{tabular}{|c|c|c|c|c|c|c|c|c|c|c|c|c|}
\hline \multirow[t]{3}{*}{ Itens alimentares } & \multirow{2}{*}{\multicolumn{2}{|c|}{$\begin{array}{l}\text { Classe } 1 \\
(\mathrm{n}=17)\end{array}$}} & \multirow{2}{*}{\multicolumn{2}{|c|}{$\begin{array}{l}\text { Classe } 2 \\
(\mathrm{n}=31)\end{array}$}} & \multirow{2}{*}{\multicolumn{2}{|c|}{$\begin{array}{l}\text { Classe } 3 \\
(n=18)\end{array}$}} & \multirow{2}{*}{\multicolumn{2}{|c|}{$\begin{array}{l}\text { Classe } 4 \\
(n=13)\end{array}$}} & \multirow{2}{*}{\multicolumn{2}{|c|}{$\begin{array}{l}\text { Classe } 5 \\
(\mathrm{n}=8)\end{array}$}} & \multirow{2}{*}{\multicolumn{2}{|c|}{$\begin{array}{c}\text { Classe } 6 \\
(\mathrm{n}=8)\end{array}$}} \\
\hline & & & & & & & & & & & & \\
\hline & FO & IA & FO & IA & FO & IA & FO & IA & FO & IA & FO & IA \\
\hline Material Vegetal & 17,6 & 0,5 & 6,4 & 0,79 & 5,5 & 25,0 & 0,97 & 0,1 & & & 25,0 & 18,8 \\
\hline Cladocera & & & 3,2 & 0,01 & & & & & & & & \\
\hline Diptera (larva/pupa) & 35,3 & 86,8 & 22,6 & 54,7 & 33,3 & 50,0 & 36,1 & 47,6 & 38,5 & 71,5 & 25,0 & 76,5 \\
\hline Coleoptera (larva) & & & & & & & & & 7,7 & 3,3 & & \\
\hline Trichoptera (larva) & 5,90 & 0,25 & & & & & & & & & & \\
\hline Restos de insetos & 5,90 & 0,25 & 6,4 & 2,8 & & & & & 7,7 & 0,5 & 12,5 & 0,36 \\
\hline Peixes & 17,6 & 12,2 & 25,8 & 41,7 & 38,9 & 25,0 & 62,9 & 52,3 & 15,4 & 24,7 & 12,5 & 4,34 \\
\hline
\end{tabular}

Fonte: Dados da pesquisa.

espécies cultivadas podem alcançar os ambientes aquáticos marginais através de (i) escapes, pela água efluente dos tanques, (ii) acidentes, por rompimento ou transbordamento dos tanques, (iii) soltura deliberada de indivíduos remanescentes nos tanques durante seu esvaziamento e (iv) descartes resultantes das atividades de manejo dos tanques (4).

Nos reservatórios da região metropolitana de Curitiba, parte da ictiofauna é composta por espécies adaptadas a ambientes lênticos, como parece ser o caso da maioria das espécies introduzidas registradas (11). Diversas espécies introduzidas têm sido registradas nos reservatório da região, destacando-se o dentudo C. stenopterus e o black-bass Micropterus salmoides, as quais perfazem mais de $23 \%$ do total capturado no Reservatório do Passaúna (3). Teoricamente, essas espécies se adaptam melhor a um reservatório, por apresentarem amplo espectro alimentar e características reprodutivas adaptadas a ambientes de águas calmas.

Com relação à alimentação, estudos sobre a dieta de peixes tropicais de água doce mostram que, de maneira geral, os peixes apresentam considerável plasticidade trófica (12), sendo quase sempre euritróficos (13), ou seja, mudam sua dieta de acordo com variações espaciais e temporais na disponibilidade dos itens alimentares. Essa flexibilidade alimentar foi registrada para C. stenopterus, o que de certa forma aumenta as chances de sobrevivência da espécie em locais com eventual escassez de recursos alimentares.

A atividade reprodutiva da espécie foi constante, atingindo picos durante as estações mais quentes do ano, coincidindo com o observado para outras espécies na Bacia do Paraná $(14,15)$. Muito embora a análise macroscópica permita falhas na eventual diferenciação de indivíduos em maturação e maduros, o longo período reprodutivo observado pode indicar um mecanismo de desova parcelada (5). Essa tática oportunista (16) é importante para o sucesso de algumas espécies nesse tipo de ambiente (reservatório), onde a manutenção de populações viáveis é garantida por um processo reprodutivo longo.

Em uma análise geral, se considerarmos o pequeno porte, a estratégia reprodutiva e o espectro alimentar, é possível caracterizarmos esta espécie como oportunista. Espécies oportunistas exibem maior facilidade para a invasão de novos ambientes (17), o que leva a crer que a abundância registrada no Reservatório do Passaúna é provavelmente decorrente da tolerância que estes organismos apresentam às variações ambientais.

As informações obtidas indicam que esta espécie introduzida pode impactar populações naturais no reservatório do Passaúna, graças à competição por alimento, abrigo ou a disseminação de parasitos (4, 
18). Esta constatação indica que medidas de manejo devem ser realizadas, como, por exemplo, a captura seletiva de exemplares, para que a conservação deste ecossistema seja alcançada.

\section{Agradecimentos}

Agradecemos a equipe do Laboratório de Pesquisa e Piscicultura (LAPEP) da Pontifícia Universidade Católica do Paraná pela realização das coletas. Ao Museu de História Natural Capão da Imbuia (MHNCI), Prefeitura Municipal de Curitiba, pelas instalações e parceria.

\section{Referências}

1. Reis RE, Kullander SO, Ferraris CJ. Check list of the freshwater fishes of South and Central America. Porto Alegre: EDIPUCRS; 2003.

2. Lucena CAS. Revisão e redefinição do gênero neotropical Charax Scopoli, 1777 com a descrição de quatro espécies novas (Pisces; Characiformes; Characidae) Comunicação do Museu de Ciências e Tecnologia da PUCRS, Série Zool. 1987;40:5-124.

3. Bastos LP, Swarofsky E, Sari C, Almada FK, Lima L, Abilhoa V, Carneiro PC. Caracterização ictiofaunística dos três reservatórios do alto Iguaçu e suas interações ambientais. Resumos do XVII Encontro Brasileiro de Ictiologia; 2007. Itajaí: Sociedade Brasileira de Ictiologia; Univalli; 2007. p. 258.

4. Agostinho AA, Julio Jr HF. Ameaça ecológica: peixes de outras águas. Ciência Hoje. 1996;21(124):36-44.

5. Vazzoler AEAM. Biologia da reprodução de peixes teleósteos: teoria e prática. Maringá: EDUEM/SBI/ CNPq/Nupelia; 1996.

6. Vieira S. Introdução à bioestatística. Rio de Janeiro: Campus; 1980.

7. Hynes HBN. The food of fish-water sticklebacks (Gasteronotus aculeatus and Pygosteus pungitius), with a review of methods used in studies of the food of fishes. J. Anim Ecol. 1950;19:36-57.

8. Hyslop EJ. Stomach contents analisys: a review of methods and their application. J Fish Biol. 1980;17(4):411-29.
9. Kawakami E, Vazzoler G. Método gráfico e estimativa deíndice alimentar aplicado no estudo de alimentação de peixes. Bolm Inst Oceanogr. 1980;29(2):205-7.

10. Welcomme RL. Internacional introductions of inland aquatic species. Rome: FAO Fish Tec Papers. 1988;(294):1-318.

11. Abilhoa V. Composição, aspectos biológicos e conservação da ictiofauna do alto curso do rio Iguaçu, Região Metropolitana de Curitiba, Paraná, Brasil [tese]. Curitiba, Universidade Federal do Paraná; 2004.

12. Lowe-McConnell RH. Ecological studies in tropical fish communities. Cambridge: Cambridge Univ. Press; 1987.

13. Abelha MCF, Agostinho AA, Goulart E. Plasticidade trófica em peixes de água doce. Acta Scientiarum. 2001;23(2):425-34.

14. Vazzoler AEAM, Menezes NA. Síntese dos conhecimentos sobre o comportamento reprodutivo dos Characiformes da América do Sul (Teleostei, Ostariophysi). Rev Bras Biol. 1992;52(4):626-40.

15. Agostinho AA, Hahn NS, Vazzoler AEAM. A planície de inundação do alto rio Paraná. Maringá: EDUEM; Nupélia; 1997.

16. Winemiller KO. Paterns of variation in life history among South American fishes in seasonal environments. Oecologia. 1989;81:225-41.

17. Agostinho AA, Gomes LC. Manejo e monitoramento de recursos pesqueiros: perspectivas para o reservatório de Segredo. In: Agostinho AA, Gomes LC. Reservatório de Segredo: bases ecológicas para o manejo. Maringá: EDUEM; 1997. p. 106-121.

18. Agostinho AA. O lado oculto da introdução de peixes. Boletim Informativo da Abrapoa 1996;9(1).

Recebido: 03/04/2009 Received: 04/03/2009

Aprovado: 14/10/2009 Approved: 10/14/2009 